ARTICLE

DOI: $10.1038 / s 41467-017-01431-5$

\title{
piRNAs and Aubergine cooperate with Wispy poly(A) polymerase to stabilize mRNAs in the germ plasm
}

Jérémy Dufourt ${ }^{1}$, Gwénaëlle Bontonou', Aymeric Chartier ${ }^{1}$, Camille Jahan', Anne-Cécile Meunier ${ }^{1}$, Stéphanie Pierson ${ }^{1}$, Paul F. Harrison (1) ${ }^{2,3}$, Catherine Papin ${ }^{1}$, Traude H. Beilharz ${ }^{3} \&$ Martine Simonelig (D) ${ }^{1}$

Piwi-interacting RNAs (piRNAs) and PIWI proteins play a crucial role in germ cells by repressing transposable elements and regulating gene expression. In Drosophila, maternal piRNAs are loaded into the embryo mostly bound to the PIWI protein Aubergine (Aub). Aub targets maternal mRNAs through incomplete base-pairing with piRNAs and can induce their destabilization in the somatic part of the embryo. Paradoxically, these Aub-dependent unstable mRNAs encode germ cell determinants that are selectively stabilized in the germ plasm. Here we show that piRNAs and Aub actively protect germ cell mRNAs in the germ plasm. Aub directly interacts with the germline-specific poly(A) polymerase Wispy, thus leading to mRNA polyadenylation and stabilization in the germ plasm. These results reveal a role for piRNAs in mRNA stabilization and identify Aub as an interactor of Wispy for mRNA polyadenylation. They further highlight the role of Aub and piRNAs in embryonic patterning through two opposite functions.

\footnotetext{
${ }^{1}$ mRNA Regulation and Development, Institute of Human Genetics, UMR9002 CNRS-University of Montpellier, 141 rue de la Cardonille, 34396 Montpellier Cedex 5, France. ${ }^{2}$ Monash Bioinformatics Platform, Monash University, Melbourne, VIC 3800, Australia. ${ }^{3}$ Development and Stem Cells Program, Monash Biomedicine Discovery Institute, Monash University, Melbourne, VIC 3800, Australia. Catherine Papin Deceased. Correspondence and requests for materials should be addressed to M.S. (email: Martine.Simonelig@igh.cnrs.fr)
} 
G erm granules are specific ribonucleoprotein granules found in germ cells of all species. They contain mRNAs that have essential functions in germ cell specification and/or development ${ }^{1}$. In Drosophila, the germ plasm starts to assemble during mid-oogenesis with the localization and translation, at the posterior pole of the oocyte, of oskar (osk) mRNA that encodes the primary determinant of the germ plasm. Other maternal mRNAs then localize to the germ plasm using different mechanisms ${ }^{2}$. First, mRNA localization involves a diffusion and anchoring mechanism taking place at late stages of oogenesis, during nurse cell dumping, when nurse cells empty their content into the oocyte $\mathrm{e}^{3,4}$. This mechanism is very inefficient, resulting in germ plasm localization of only $4 \%$ of maternal nanos (nos) mRNA, which encodes a conserved major germline determinant ${ }^{5,6}$. A second mechanism takes place in the early embryo to complete mRNA localization, and involves mRNA decay and selective stabilization in the germ plasm $^{7}$. The molecular basis underlying the link between mRNA decay and mRNA localization to the germ plasm has remained elusive.
Components of the Piwi-interacting RNA (piRNA) pathway, including Vasa and the PIWI protein Aubergine (Aub) are core components of germ granules ${ }^{8}$, suggesting a potential link between the piRNA pathway and mRNA regulation in germ granules. piRNAs are a class of small 23-30 nucleotides (nt) RNAs bound to specific Argonaute proteins, the PIWI proteins. They are involved in the repression of transposable elements (TEs) in the germline ${ }^{9,10}$. piRNAs loaded into the cytoplasmic PIWI proteins Aub and Argonaute 3 (Ago3) target TE mRNA sequences through complementarity and guide their cleavage by the endonucleolytic activity of Aub and Ago3.

The more recent data have demonstrated the role of the piRNA pathway in post-transcriptional gene regulation ${ }^{11-17}$. In Drosophila, piRNAs produced in the female germline are provided maternally to the embryo, mostly loaded into Aub. Aub is present both in the somatic part of the embryo and at higher levels in the posterior germ plasm ${ }^{15}$. We previously showed that Aub targets maternal mRNAs through incomplete base-pairing with piRNAs and can induce their destabilization by either direct cleavage, or
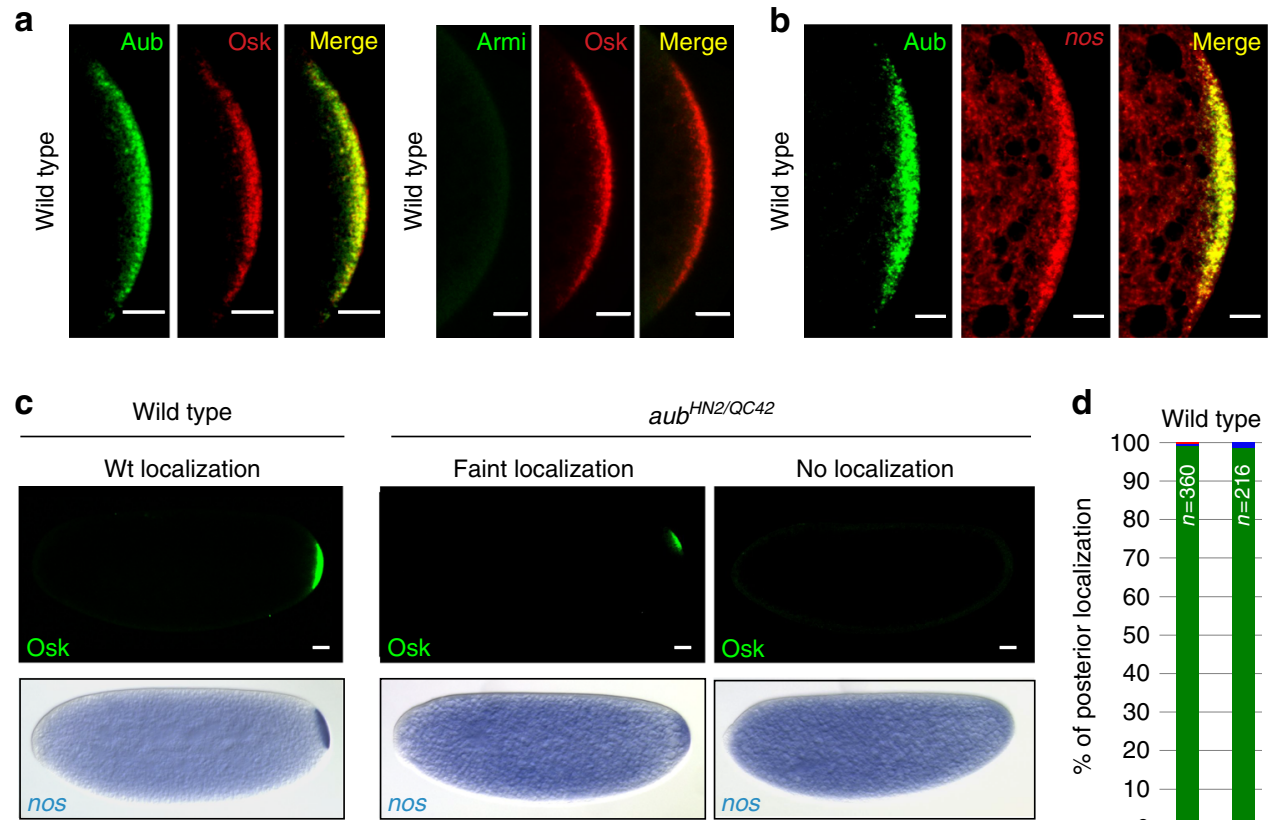

$m n k^{p 6} a u b^{H N 2 /} m n k^{p 6} a u b^{Q C 42}$
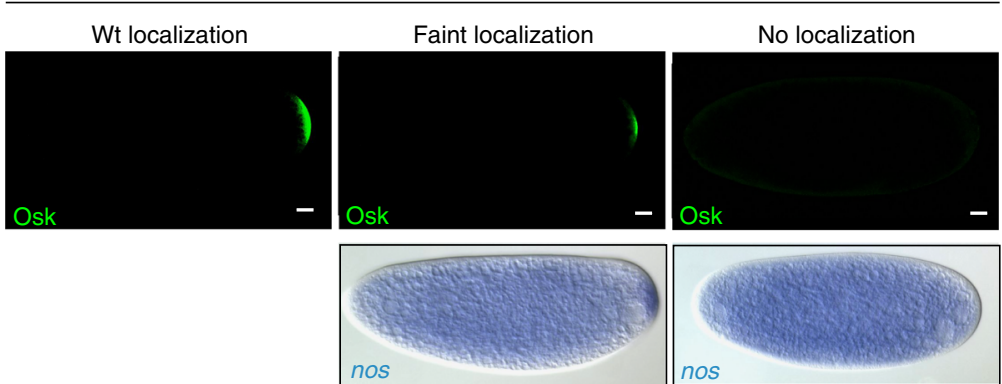

d

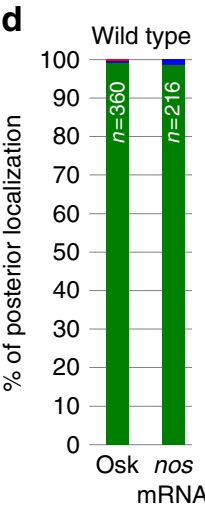

\section{Colocalization

Aub in
nos mRNA


a

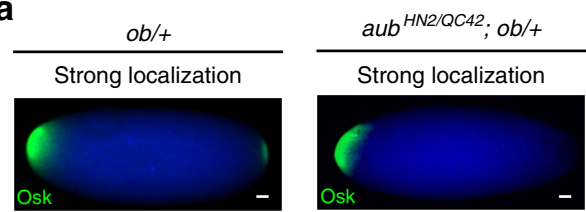

b
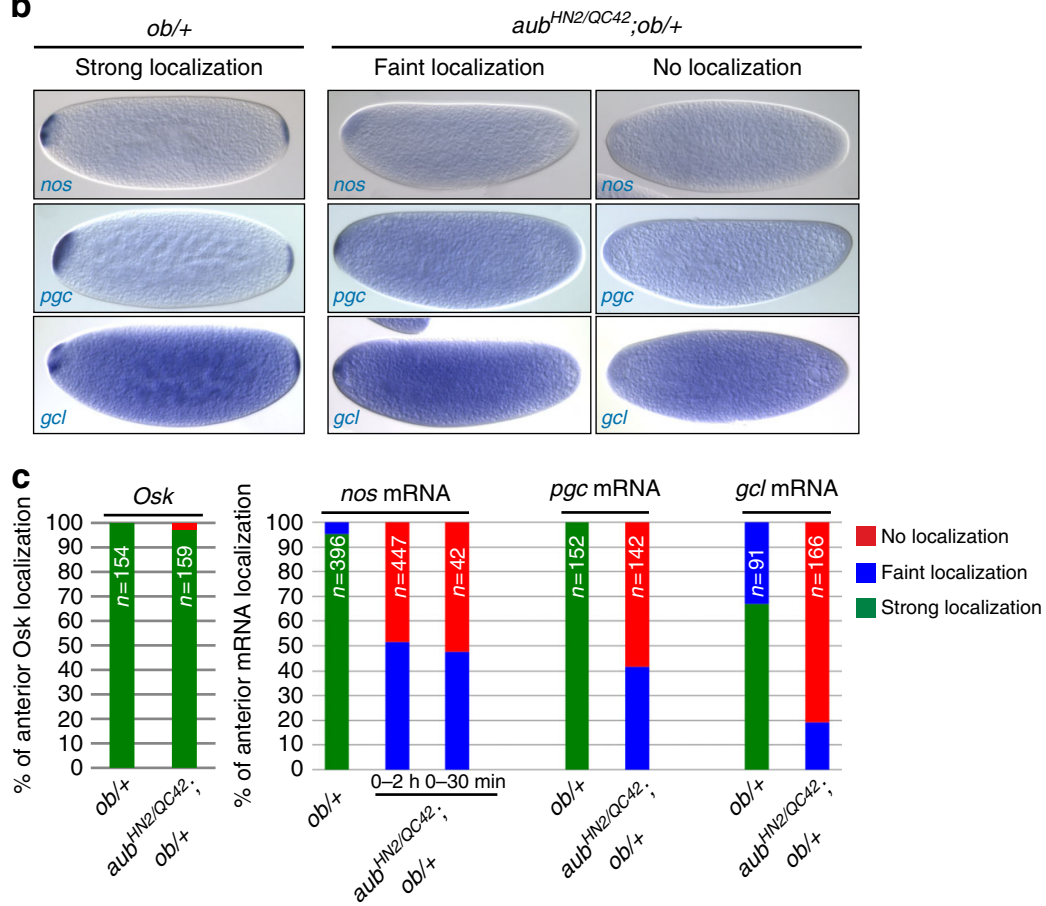

d

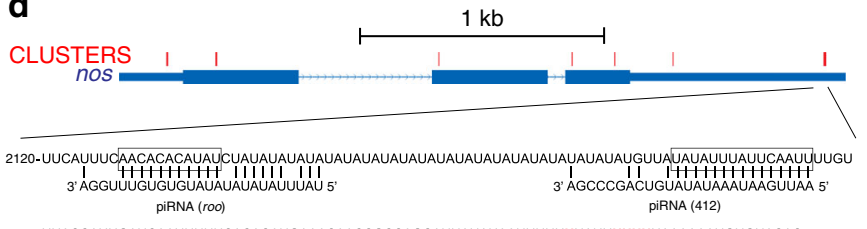

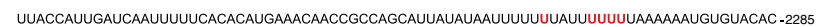

e

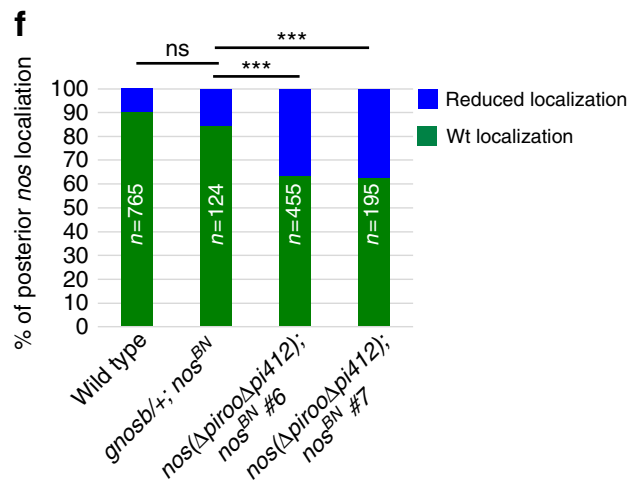

Fig. 2 Role of Aub and piRNAs in germ cell mRNA localization to the germ plasm. a Immunostaining with anti-Osk of osk-bcd3'UTR (ob) embryos in wildtype or aub mutant backgrounds. The DAPI staining background (blue) shows the bulk of the embryo. Scale bars: $30 \mu \mathrm{m}$. $\mathbf{b}$ In situ hybrydization of $0-2 \mathrm{~h}$ $o b /+$ embryos either in wild-type or aub mutant backgrounds, with nos, pgc and gcl RNA probes. c Quantification of Osk and mRNA localization shown in $\mathbf{a}, \mathbf{b}$, respectively. 0-30min-embryos were also quantified for nos mRNA. $\mathbf{d}$ Schematic representation of nos mRNA and base-pairing with piRNAs. Thin boxes are 5'- and 3'-UTRs, lines are introns, and thick boxes are exons. Crosslink clusters from Aub-iCLIP are indicated in red. The sequence of the region with the strongest crosslink sites is shown. Base-pairing with representative piRNAs from roo and 412 TEs is shown; the deletions overlaping the piRNA target sites in the nos ( $\Delta$ piroo $\Delta$ pi412) transgene are boxed ${ }^{15}$. Aub-crosslinked nt are in red. e nos mRNA in situ hybrydization of $0-2 \mathrm{~h}$-embryos from wildtype and nos( $\Delta$ piroo $\Delta$ pi412); nos ${ }^{B N}$ females. The nos ${ }^{B N}$ mutant does not produce nos mRNA in the embryo. $\mathbf{f}$ Quantification of nos mRNA posterior localization as shown in $\mathbf{e}$, for wild-type embryos, nos ${ }^{B N}$ embryos bearing the wild-type genomic nos (gnosb) transgene, and nos ${ }^{B N}$ embryos bearing the nos ( $\Delta$ piroo $\Delta$ pi412) transgene from two independent stocks. ns: non-significant, ${ }^{\star \star \star} p<0.001$, using $\chi 2$ test 
the recruitment of the CCR4-NOT deadenylation complex together with the RNA-binding protein Smaug (Smg) ${ }^{11,15}$. Strikingly, Aub-dependent unstable mRNAs that encode germ cell determinants, undergo selective stabilization: these mRNAs are degraded in the somatic part of the embryo, while they are stabilized and translated in the germ plasm to participate in germ cell development ${ }^{11}$. This raises the question of the role of piRNAs and Aub in the stabilization of these germ cell mRNAs in the germ plasm. Here we show, using nos mRNA as a paradigm, that piRNAs and Aub play an active role in the protection of germ cell mRNAs in the germ plasm.

Here we find that the germline-specific non-canonical poly(A) polymerase Wispy (Wisp) is a direct interactor of Aub, which colocalizes with Aub in the germ plasm. Furthermore using a method for poly $(\mathrm{A})$ tail sequencing, we uncover the role of Aub in polyadenylation of a pool of germ cell mRNAs that have long poly(A) tails. We conclude that Aub acts by directly recruiting Wisp to germ cell mRNAs, leading to their polyadenylation and stabilization in the germ plasm. These results reveal a role for piRNAs in mRNA stabilization. They further identify a critical role of Aub and piRNAs in embryonic patterning through two opposite functions: somatic decay and germline stabilization of germ cell mRNAs, thus revealing the molecular link between these two processes.

\section{Results}

mRNA localization in germ plasm depends on Aub and piRNAs. We have previously shown that Aub directly binds nos mRNA and is required for its deadenylation and decay in the somatic part of the early embryo ${ }^{11,15}$. However, nos mRNA also colocalizes with Aub protein in the germ plasm and primordial germ cells, where it is stabilized ${ }^{18}$. This suggests a different function of Aub in these regions. We used $a u b$ and armitage (armi) mutants to address a potential active role of Aub and piRNAs in nos mRNA localization in the germ plasm. Note that "mRNA localization" is used throughout, independently of the localization mechanism involved. Armi is another component of the piRNA pathway that has a prominent role in piRNA production $^{19}$. We first validated the colocalization of Aub with nos mRNA in the germ plasm (Fig. 1a, b). Mutants of the piRNA pathway induce embryonic patterning defects through activation of the Chk2 DNA damage checkpoint. Checkpoint activation leads to defective localization of osk mRNA at the posterior pole of the oocyte and a lack of Osk protein synthesis ${ }^{20,21}$. This defect is partially rescued in double mutants for piRNA pathway components and the Chk2 kinase (mnk mutant $)^{20,21}$. Accordingly, we found that most embryos produced by $a u b^{H N 2} / a u b^{Q C 42}$ females (referred to as $a u b^{H N 2} / a u b^{Q C 42}$ embryos) and all embryos produced by $\operatorname{armi}^{1} / \mathrm{armi}^{72.1}$ females (armi ${ }^{1} / \mathrm{armi}^{72.1}$ embryos) showed a lack or very weak localization of Osk protein at the posterior pole (Fig. 1c-e, Supplementary Fig. 1b). This defective germ plasm led to a very weak or an absence of nos mRNA localization at the posterior pole in these mutant embryos. Strikingly, a wild-type localization of Osk was rescued in 40 and $15 \%$ of $m n k a u b$, and $m n k$; armi double-mutant embryos, respectively. However, wild-type nos mRNA posterior localization was not rescued (Fig. 1c-e, Supplementary Fig. 1a, b). This suggested a direct role of Aub in the localization of nos mRNA in the germ plasm. Armi did not localize to the posterior pole of the embryo (Fig. 1a), thus precluding a direct interaction between Armi and nos mRNA for nos localization at the posterior pole. Instead, strongly reduced piRNA levels in armi mutants ${ }^{19}$ could underlie the lack of nos mRNA posterior localization in these mutants. Consistent with this, we previously showed that unloaded Aub was unable to bind mRNAs and did not localize to the germ plasm ${ }^{11}$.

To confirm a direct role of Aub in mRNA localization in the germ plasm, we used the osk-bcd3'UTR $(o b)$ transgene containing the osk coding sequence followed by the bicoid $(b c d) 3^{\prime} \mathrm{UTR}$, which allows Osk localization and germ plasm formation at the anterior pole of the embryo ${ }^{22}$. Aub, as well as germ cell mRNAs were recruited and colocalized to this anterior germ plasm (Supplementary Fig. 2a) ${ }^{11}$. Although Osk localization to the anterior pole was not affected in $a u b$ mutant embryos bearing the $o b$ transgene $^{23}$ (Fig. 2a, c), nos mRNA anterior localization was lost $(48.5 \%$ of embryos) or very faint and diffuse $(51.5 \%$ of embryos) (Fig. 2b, c). A similar result was obtained for two other germ plasm mRNAs that interact with Aub, polar granule component $(p g c)$ and germ cell-less $(g c l)$ (Fig. 2b, c), which is consistent with a direct role of Aub in the recruitment of germ cell mRNAs in the germ plasm. Interestingly, anterior localization of nos mRNA was not affected in $a u b^{-} ; o b /+$ stage 10 oocytes, showing that Aub was not required at this stage (Supplementary Fig. 2b). The defects in nos mRNA localization were identical in 0-2 h- and 0-30 min-aub mutant embryos (Fig. 2c), indicating that Aub requirement for nos localization started between late oogenesis and early (0-30 min) embryogenesis.

Taken together, these results strongly suggest that Aub and piRNAs play a direct role in the localization of germ cell mRNAs in the germ plasm in late oocytes and/or early embryos.

mRNA localization in germ plasm requires targeting by piRNAs. Aub-iCLIP in embryos identified several reproducible sites of interaction between Aub and nos $\mathrm{mRNA}^{11}$. The most prominent crosslink sites were located in the distal region of nos $3^{\prime}$ UTR (Fig. 2d), and deletion of two putative piRNA target sites (from roo and 412 TEs) there led to defective nos mRNA deadenylation in the somatic part of the embryo ${ }^{15}$. We propose that the same piRNA-guided Aub interactions with nos mRNA result in deadenylation and decay in the soma, and localization in the germ plasm. We, thus, analyzed the localization of nos mRNA deleted for these two piRNA target sites. Posterior localization was significantly reduced in 36 and $37 \%$ of embryos in two independent nos $\left(\Delta\right.$ piroo $\Delta$ pi412); nos ${ }^{B N}$ transgenic stocks, compared to localization of nos mRNA from a wild-type genomic transgene $^{24}$ (Fig. 2e, f). This localization defect was not due to reduced expression of the nos $(\Delta$ piroo $\Delta$ pi412) transgene (Supplementary Fig. 2c). The fact that nos localization defect was weaker for nos $(\Delta$ piroo $\Delta$ pi412) transgene than in $a u b$ mutant embryos, was expected since several Aub-binding sites remained unaffected in this transgene (Fig. 2d). Nonetheless, this reduced localization following the removal of only two piRNA target sites argues that Aub binding to at least a number of sites in mRNAs, depends on sequence-specific targeting by piRNAs. These results indicate that mRNA binding by Aub does not only rely on random piRNA targeting along the entire length of mRNAs through very low complementarity, as was proposed previously ${ }^{25}$ Consistent with this, using Aub-iCLIP data sets ${ }^{11}$, we found that the proportion of mRNAs potentially targeted by piRNAs with high complementarity was significantly higher in Aubcrosslinked than in non-crosslinked mRNAs (Supplementary Fig. 2d). In addition, we found a strong overlap between Aubcrosslinked mRNAs and mRNAs producing piRNAs upon targeting by a highly complementary trigger piRNA ${ }^{26}$ (Supplementary Fig. 2e).

These results are consistent with a role of piRNA sequencespecific targeting for Aub interaction with mRNAs and their localization to the posterior germ plasm. 
Aub methylation in both soma and germ plasm. The dual role of Aub in mRNA decay in the soma and localization in the germ plasm indicates a switch in Aub function between these two regions of the embryo. Aub undergoes symmetric arginine dimethylation by the protein arginine methyltransferase 5,
Capsuleen (Csul) ${ }^{27}$. Aub methylation is required for its interaction with Tudor (Tud) and its localization to the germ plasm ${ }^{27,28}$. We asked whether arginine dimethylation could participate in the switch in Aub function. In this hypothesis, only the pool of Aub localized in the germ plasm would be methylated, mRNA decay a

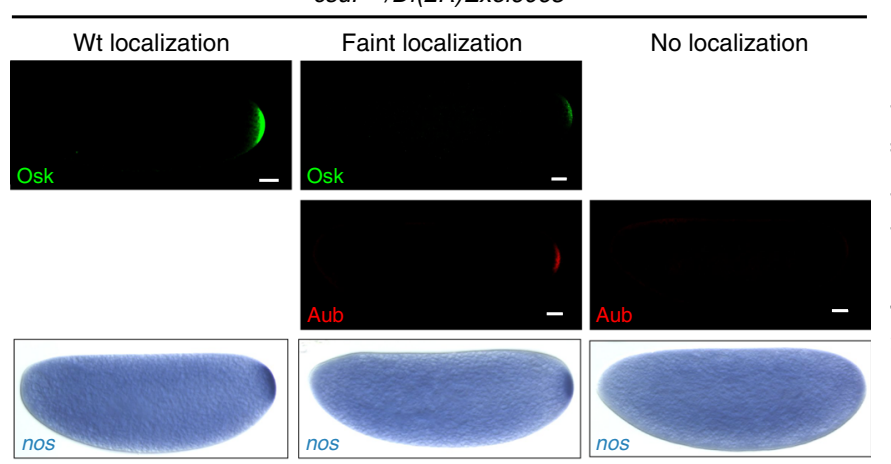

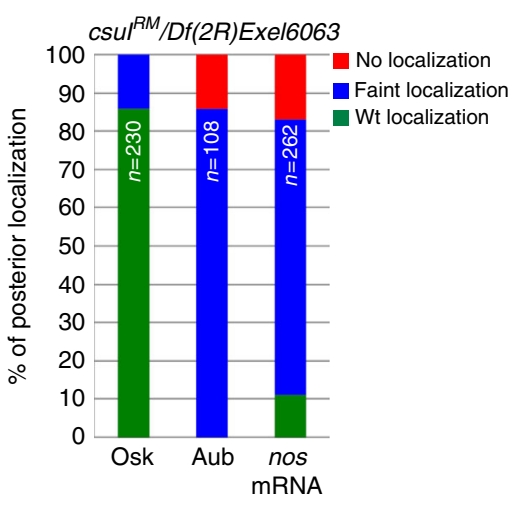

b

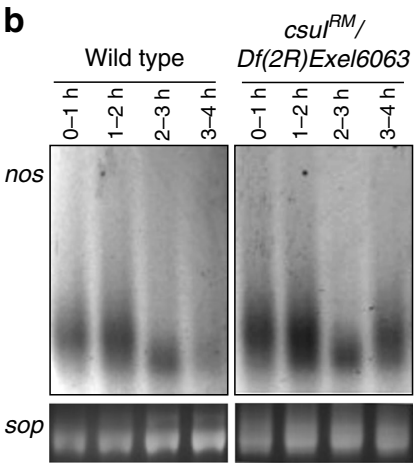

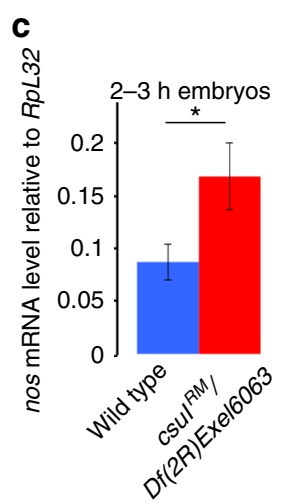

d

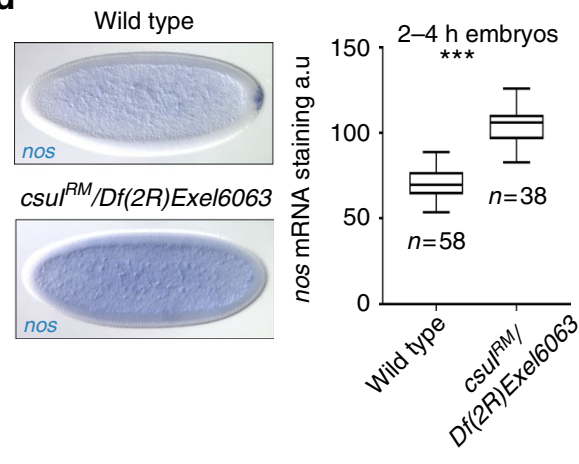

e

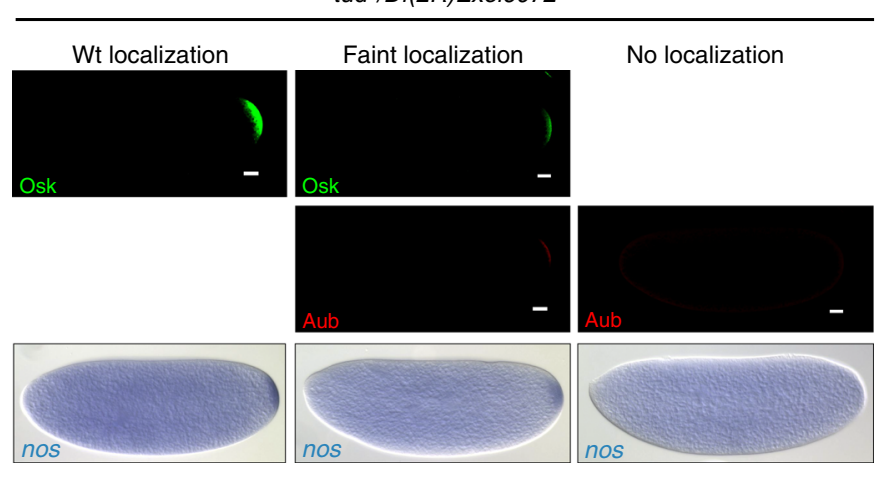

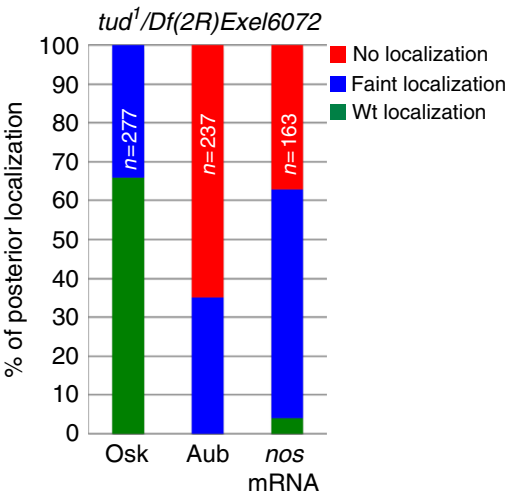
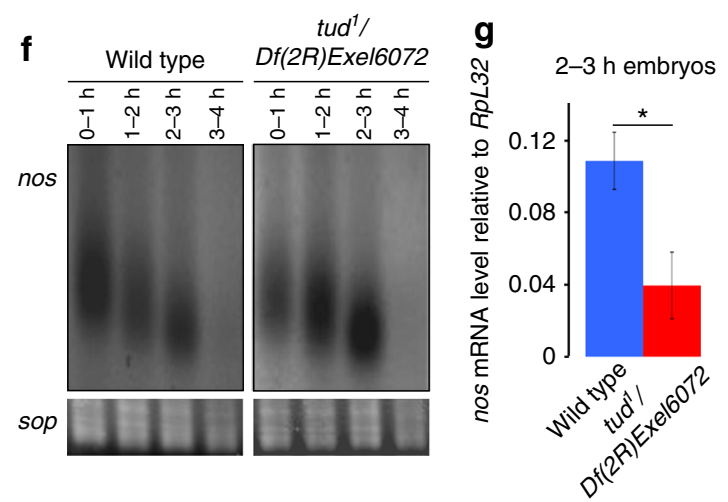

h

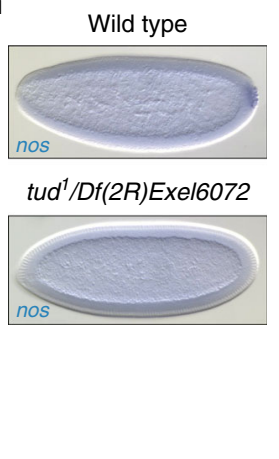

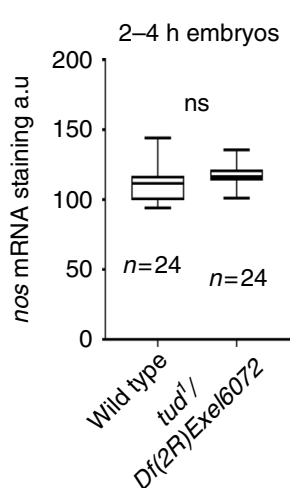


in the soma would involve unmethylated Aub. We analyzed both Aub functions when arginine dimethylation was defective in csul mutants. Posterior accumulation of both Aub and nos mRNA were strongly affected in csul mutant embryos (Fig. 3a, Supplementary Fig. 3a). We used poly(A) test (PAT) assays to record nos mRNA deadenylation in the bulk of csul mutant embryos during the first $4 \mathrm{~h}$ of embryogenesis. nos mRNA deadenylation profile was affected in mutant embryos, with long poly $(\mathrm{A})$ tails still present at 3-4 h of development (Fig. 3b). This deadenylation defect correlated with stabilized nos mRNA in csul mutant embryos, both quantified by RT-qPCR and visualized in the soma by in situ hybridization (Fig. 3c, d). Consistent with impaired mRNA decay, a large proportion of csul mutant embryos did not hatch (Supplementary Fig. 3b).

Tud protein is restricted to the germ plasm in the embryo and is required for Aub posterior localization ${ }^{29}$. As expected, Aub and nos mRNA posterior localization was strongly affected in tud mutant embryos (Fig. 3e). In contrast, Aub-dependent somatic nos mRNA deadenylation and decay were not reduced in tud mutant embryos (Fig. 3f-h) and most of these embryos hatched (Supplementary Fig. 3b). Using embryo immunostaining with SYM11 antibody that specifically recognizes symmetric dimethylarginines, we confirmed the defect of arginine dimethylation in csul mutant embryos (Supplementary Fig. 3c). In tud mutant embryos, arginine dimethylation level was not reduced, but arginine dimethylated proteins did not accumulate in the germ plasm (Supplementary Fig. 3c).

These results indicate that both the somatic and germline pools of Aub are methylated. They further show that Aub arginine dimethylation is required for both Aub functions in mRNA decay and localization in the germ plasm. Therefore, this posttranslational modification is not responsible for the switch in Aub function between soma and germ plasm.

Aub recruits Wisp to stabilize mRNAs in the germ plasm. Wisp is the germline-specific poly(A) polymerase involved in cytoplasmic polyadenylation of a large number of mRNAs during late oogenesis and early embryogenesis ${ }^{30-34}$. Since Wisp is required for stabilization and posterior localization of osk and nos mRNAs in embryos ${ }^{30}$, we asked whether it could cooperate with Aub in mRNA stabilization and localization in the germ plasm. Wisp accumulated in the germ plasm in the oocyte where it strongly colocalized with Aub (Fig. 4a, b). Wisp was present in the whole embryo with higher accumulation in the germ plasm (Fig. $4 \mathrm{~b}$ ). It substantially colocalized with Aub in this region, as well as in primordial germ cells (Fig. 4b, Supplementary Fig. 4a). In addition, Wisp was recruited to the anterior germ plasm and colocalized with Aub in embryos expressing the ob transgene (Supplementary Fig. 4b). Analysis of Wisp-Aub colocalization in the germ plasm in csul and tud mutant oocytes showed that it was maintained in these mutant backgrounds, indicating that Wisp colocalization with Aub did not require Aub arginine dimethylation (Supplementary Fig. 4c). Strikingly, the levels of Wisp in the germ plasm correlated with the lower levels of posteriorly localized Aub in these mutants, which is consitent with a role of Aub in the recruitment of Wisp to the germ plasm (Supplementary Fig. 4c). In contrast, the CCR4 deadenylase was depleted and Smg foci were smaller in the germ plasm where Aub accumulated (Fig. 4c).

We used co-immunoprecipitation in $0-2 \mathrm{~h}$-embryos to show that Wisp coprecipitated with Aub, independently of the presence of RNA (Fig. 4d). The reverse experiment confirmed the coprecipitation of Aub with Wisp, in the absence of RNA, in 0-2 h-embryos (Fig. 4e). Moreover, this coprecipitation was maintained in $0-2 \mathrm{~h}-$ csul and -tud mutant embryos (Fig. 4e), revealing that the Aub/ Wisp complex was independent of Aub arginine dimethylation, and could form in the somatic part of the embryo, since the levels of localized Aub were low in these mutant embryos.

Direct interactions between Aub and Wisp were analyzed using GST pull-down experiments. Aub contains three domains specific of Argonaute proteins (PAZ, MID and PIWI) (Fig. 4f). In vitrotranslated HA-tagged Aub(1-482), which contained the PAZ domain, bound to recombinant GST-Wisp(1-713) and GSTWisp(702-1373), but not to GST-Wisp(11-547) or GST alone (Fig. 4f). Wisp recombinant proteins that interacted with HAAub(1-482) overlapped over the central region of Wisp; we thus used GST-Wisp(636-746) to validate that the central region of Wisp interacted with HA-Aub(1-482) (Fig. 4f). In contrast, HAAub(476-866) that contained the MID and PIWI domains did not bind to any of the GST-Wisp proteins (Fig. 4f). These results reveal direct interactions between the central part of Wisp and the N-terminal half of Aub.

We used PAT assays and mPAT, a method for digital PAT assays multiplexed for high-throughput sequencing, to address a role of Aub in poly(A) tail elongation of nos mRNA localized in the germ plasm. Aub is required for deadenylation of the vast majority of nos mRNA (96\%) present in the somatic region of the embryo ${ }^{15}$. Therefore, we used $o b$-expressing embryos to increase the pool of nos mRNA localized in the germ plasm. PAT assays from these embryos at $0-1 \mathrm{~h}$ and $1-2 \mathrm{~h}$ of development identified two pools of nos mRNA with poly(A) tails of $\sim 12-60 \mathrm{nt}$, and 80 to $130 \mathrm{nt}$, respectively (Fig. 5a). In the wild-type background, poly (A) tail length of the pool with shorter poly $(\mathrm{A})$ tails decreased in size with time by deadenylation, as previously reported ${ }^{7}$. In $a u b$ mutant embryos expressing $o b$, deadenylation of the pool with shorter poly $(\mathrm{A})$ tails was reduced, and the pool with longer poly (A) tails completely disappeared (Fig. 5a). mPAT, which uses Illumina MiSeq based sequencing to identify and quantify PAT amplicons, confirmed these effects on both pools of nos mRNA in $a u b$ mutant embryos (Fig. 5b). In addition, similar effects were observed for $p g c$ mRNA, but not for the control mRNA tim10, which was not bound by Aub (Supplementary Fig. 5a, b). Because nos mRNA deadenylation in the somatic region was not strongly affected in tud mutant embryos, we sequenced nos poly(A) tails in these embryos using mPAT, to address a role of posteriorly localized Aub in nos mRNA polyadenylation in the germ plasm. Strikingly, nos mRNA poy(A) tails longer than $80 \mathrm{nt}$ were specifically shortened in tud mutant embryos at both time points (0-1 h and 1-2h), consistent with the function of Aub in the

\footnotetext{
Fig. 3 Role of Csul methyltransferase and Tud for Aub functions in mRNA decay and localization. a, e Immunostaining with anti-Osk and anti-Aub, and nos mRNA in situ hybrydization of 0-2 h-csul (a) and -tud (e) mutant embryos. Quantifications of posterior localization in embryos shown in a, e are indicated (right panels). Scale bars: $30 \mu \mathrm{m}$. b, f PAT assays of nos mRNA in embryos at $1 \mathrm{~h}$-intervals up to $4 \mathrm{~h}$ of development in wild type, csul (b) and tud (f) mutants. sop was used as a control mRNA. c, g nos mRNA quantification using RT-qPCR in 2-3 h wild-type, csul (c) and tud (g) mutant embryos. Normalization was with RpL32 mRNA. For each genotype, mRNA levels at 2-3 h were normalized to the levels at 0-1 h. Means are from three biological replicates. The error bars represent SD. ${ }^{\star} p<0.05$ using the two-tailed Student's $t$ test. d, h nos mRNA in situ hybrydization of $2-4 \mathrm{~h}$ wild-type, csul (d) and tud (h) mutant embryos. Box plots showing the quantification of nos mRNA stabilization in the somatic part of the embryo. The central horizontal bar represents the median. ns: non-significant, ${ }^{\star \star \star} p<0.001$, using the two-tailed Student's $t$ test
} 
germ plasm for nos polyadenylation (Fig. 5c). Similar effects were recorded on $p g c$ mRNA, but not the control tim10 mRNA, sequenced using mPAT (Supplementary Fig. 5c).

Taken together, these results identify the role of Aub in the germ plasm for the recruitment of Wisp to germ cell mRNAs and their polyadenylation.

\section{Discussion}

A number of recent studies have reported the role of piRNAs in cellular mRNA regulation, in addition to their role in repressing transposable elements. mRNA regulation by piRNAs is required for sex determination in Bombyx mori and embryonic patterning in Drosophila ${ }^{11,14,15}$. Furthermore, piRNAs are also involved in a

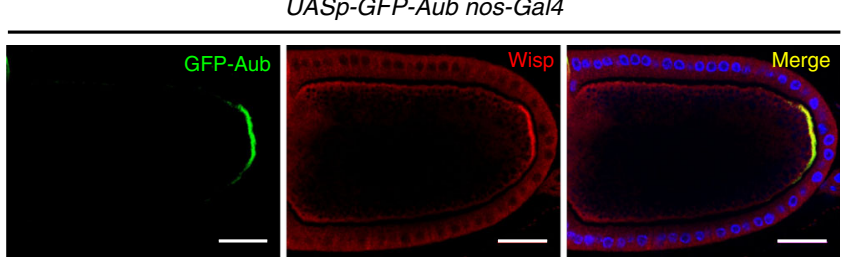

UASp-GFP-Aub nos-Gal4

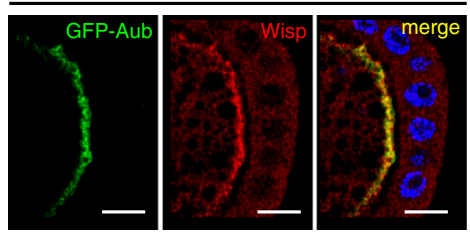

C UASp-GFP-Aub/nos-Gal4

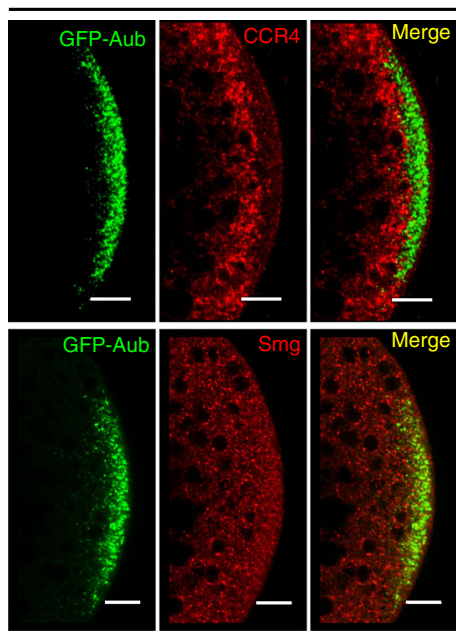

b

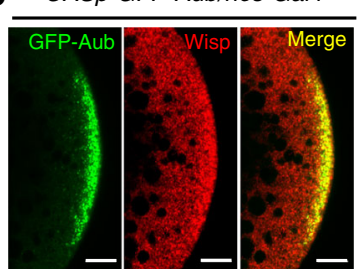

Oocyte Embryo

Colocalization Aub in Wispy

d

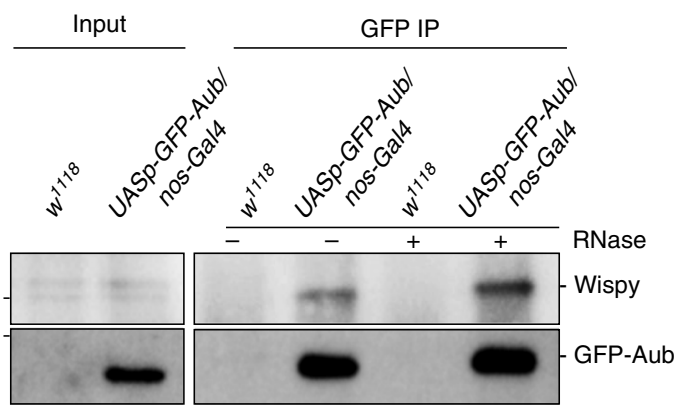

e

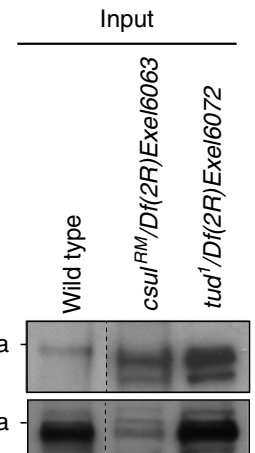

Wispy IP (+ RNase)
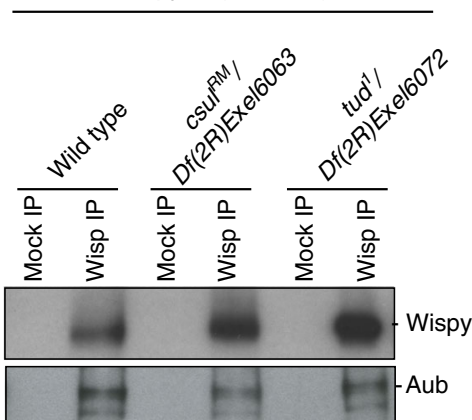

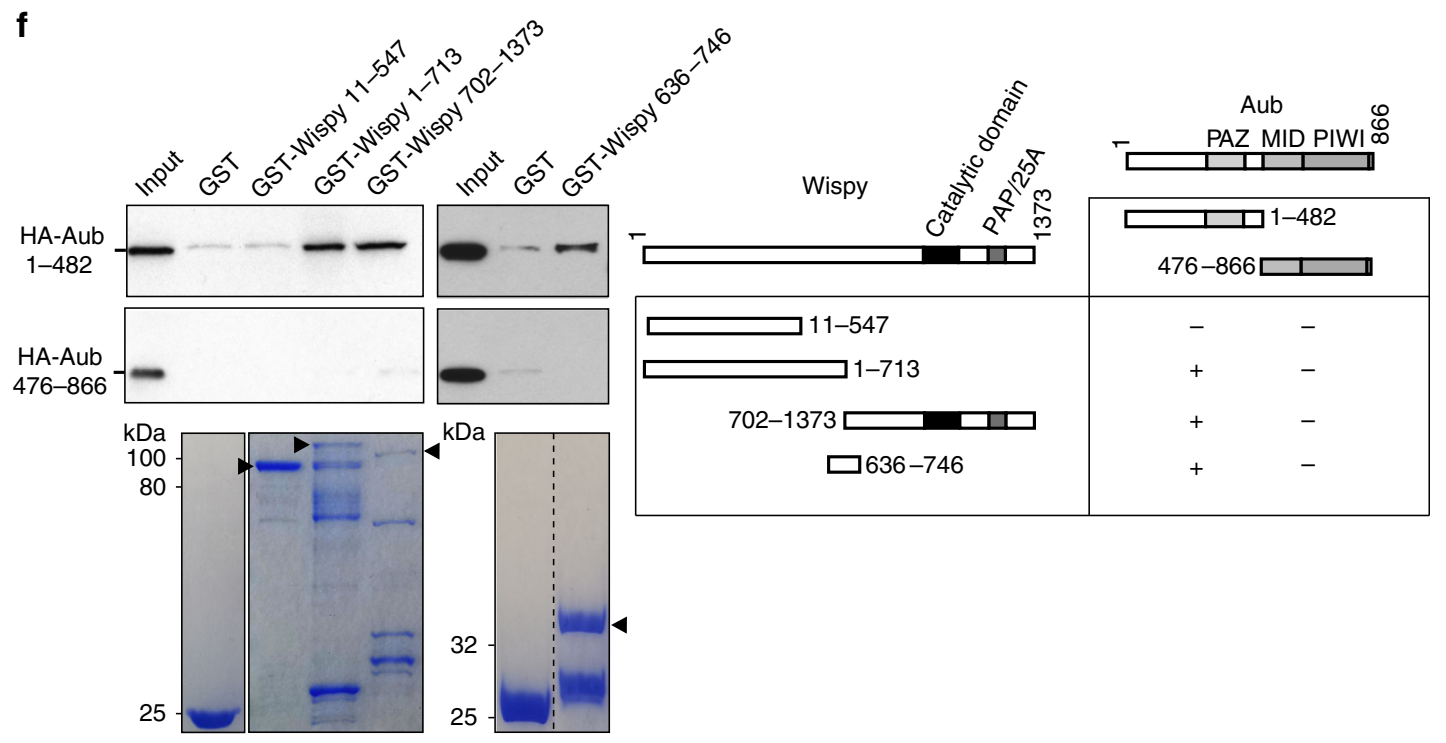


the decay of a large number of mRNAs and long non-coding RNAs during mouse sperm development ${ }^{13,16}$. mRNA basepairing with piRNAs leads to their decay either by cleavage through the endonuclease activity of PIWI proteins, or by deadenylation ${ }^{11-13,15-17}$. Cleavage of cellular transcripts upon piRNA targeting produces sense piRNAs from these transcripts by ping-pong and phasing, thus unambigously identifying mRNAs subject to this regulation ${ }^{12,26}$.

Here we identify a mechanism of piRNA-dependent regulation that results in mRNA stabilization. Aub interacts with several hundred maternal mRNAs and induces their decay during the maternal-to-zygotic transition in the embryo ${ }^{11}$. Importantly, Aub-dependent destabilized mRNAs are locally stabilized in the germ plasm and encode germ cell determinants. A link has been described between maternal mRNA decay and posterior localization, in the embryo ${ }^{35}$. We propose that Aub binding to these mRNAs and its dual role in somatic decay and stabilization in the germ plasm might be the basis for this link (Fig. 5d). A role of Aub in germ cell mRNA posterior localization has been hypothezised based on Aub interaction with these mRNAs ${ }^{25}$, and Aub has been reported to be a component of the nos mRNA localization complex ${ }^{36}$. Here we decipher the molecular mechanisms underlying this Aub function.

We identify a key component of the switch in Aub function between soma and germline. Aub directly interacts with the Wisp poly(A) polymerase, thus allowing poly(A) tail elongation and stabilization of germ cell mRNAs in the germ plasm. Osk is likely a major actor of mRNP-complex remodeling in the germ plasm, leading to Aub functional switch and Wisp activity (Fig. 5d). Osk colocalizes with Aub and Tud in germ granules ${ }^{6}$. Osk also directly interacts with Smg and prevents Smg interaction with nos mRNA, thus precluding its deadenylation and decay in the germ plasm $^{7,37}$. Consistent with this, we show that in the germ plasm, Smg granules undergo remodeling and CCR4 deadenylase is depleted. The role of Aub and Wisp in polyadenylation points to their function in mRNA localization through selective stabilization in the germ plasm. However, a role of Aub and Wisp in the localization mechanism involving posterior anchoring during late oogenesis is also possible since Aub interacts with mRNAs in ovaries $^{25}$. In addition, defects in mRNA anchoring occur in wisp mutants, suggesting a potential role of Wisp or mRNA poly(A) in anchoring ${ }^{30}$.

The presence of Aub and Wisp in the same complex in tud mutant embryos, in which Aub localization in the germ plasm is very low, suggests the possible interaction of both proteins in the somatic region. Thus, Wisp might be present in a complex containing Aub, Smg and CCR4-NOT in the soma; however, its activity would be repressed by other components in this complex. This repression would be relieved in the germ plasm through the presence of Osk and the loss of Smg from the complex (Fig. 5d).

Like all members of the GLD-2 family of poly(A) polymerases, Wisp does not bind RNA but relies on RNA-binding proteins for its recruitment to mRNAs ${ }^{38}$. We identify Aub as an RNA-binding protein involved in Wisp interaction with mRNAs in embryos. Intriguingly, Aub mode of mRNA binding that depends on diverse piRNAs is consistent with the lack of specific motifs in Wisp mRNA targets ${ }^{32,34}$. Poly(A) tail sequencing at the genomic scale has recently been used to identify mRNAs undergoing Wisp-dependent cytoplasmic polyadenylation in early embryos $^{33,34}$. Comparison of these mRNAs to Aub-interacting mRNAs in embryos ${ }^{11}$ showed that up to $25 \%$ of Wisp target mRNAs were also bound by Aub (Supplementary Fig. 5d), indicating a widespread role of Aub in Wisp-dependent cytoplasmic polyadenylation.

Several studies addressing nos mRNA posterior localization reported the role of discrete, but partially redundant localization elements $^{4,36}$. Our data are consistent with these findings: They propose the involvement of piRNA target sites highly complementary to piRNAs for mRNA localization. Deletion of these sites in nos mRNA induces only partial mislocalization, indicating redundancy with other localization elements.

These data reveal a role for piRNAs and PIWI proteins in mRNA stabilization, and uncover a major developmental function of piRNAs in germ cell specification. They further highlight the central role of Aub in coupling piRNA inheritance and mRNA regulation for germ cell development and maintenance through generations.

\section{Methods \\ Drosophila stocks and genetics. The $w^{1118}$ stock was used as a control. Mutant stocks were $a u b^{H N 2} \mathrm{cn}^{1} b w^{1} / C y O$, aub $b^{Q C 42} \mathrm{cn}^{1} b w^{1} / C y O^{39}, w^{*} ;$ armi $i^{72.1} / T M 6 C, y^{1}$ $w^{*} ;$ P $\{$ lacW $\}$ armi $^{1} / T_{M 3}{ }^{40}, m_{n} k^{P 641}, m n k^{P 6} a u b^{H N 2} / C y O, m n k^{P 6} a u b^{Q C 42} / C y O$, $m n k^{P 6}$; armi ${ }^{1} / S M 6-T M 6 B, m_{n}{ }^{P 6}$; armi $^{72.1} /$ SM6-TM6B $^{20}$, tud bw $^{1} s p^{1} / C y O^{42}$, $w^{1118} ; D f(2 R)$ Exel6072/CyO that overlaps $t u d^{43}, c s u l^{R M} / C y O^{44}, w^{1118} ; D f(2 R)$ Exel6063/CyO that overlaps csul ${ }^{43}$, nos ${ }^{B N} / T M 3^{45}, y^{1}$ wisp ${ }^{K G 05287} / F M 7 c^{30}$ and $D f(1)$ $R A 47 / F M 7 c$ that overlaps wisp. Transgenic stocks were osk-bcd3'UTR (ob21 and ob42 on second chromosome and $o b 31$ on third chromosome) ${ }^{22}$, nos-Gal4:VP16 ${ }^{46}$ $U A S p-G F P-A u b^{47}$, nos( $\Delta$ piroo- $\Delta$ pi412) ${ }^{15}$, and gnosb (wild-type genomic nos transgene $)^{24}$}

Immunostaining and RNA in situ hybridization. Embryos were dechorionated with bleach for $3 \mathrm{~min}$ and thoroughly rinced with $\mathrm{H}_{2} \mathrm{O}$. They were fixed in $37 \%$ formaldehyde with heptane (1:1) for $7 \mathrm{~min}$ on a wheel; formaldehyde was replaced by methanol and embryos were vortexed for $1 \mathrm{~min}$. Embryos that sank to the bottom of the tube were rinced three times with methanol. Before immunostaining, embryos were gradually rehydrated with methanol-PBT (PBS supplemented with $0.1 \%$ Triton-X 100) and washed three times with PBT. Embryos were incubated on a wheel at room temperature twice for $30 \mathrm{~min}$ in PBT, once for $20 \mathrm{~min}$ in PBT $1 \%$ BSA, and at $4{ }^{\circ} \mathrm{C}$ overnight in PBT $1 \%$ BSA with primary antibodies. Embryos were rinced three times, washed twice for $30 \mathrm{~min}$ in PBT, then incubated in PBT 1\% BSA for $30 \mathrm{~min}$, and in PBT 1\% BSA with secondary antibodies for $2 \mathrm{~h}$ at room temperature. Embryos were rinced three times and washed twice for $30 \mathrm{~min}$ in PBT. Ovaries were dissected at room temperature in PBS, fixed with $4 \%$ paraformaldehyde, rinced and blocked with PBT containing $1 \%$ BSA for $1 \mathrm{~h}$, and incubated in PBT $1 \%$ BSA with primary antibodies overnight at $4{ }^{\circ} \mathrm{C}$. Ovaries were washed three times in PBT 1\% BSA for $10 \mathrm{~min}$ at room temperature. They were incubated in PBT $0.1 \%$ BSA with secondary antibodies for $2 \mathrm{~h}$ at room temperature, then washed three times in PBT for $10 \mathrm{~min}$. DNA staining was performed using DAPI at $0.5 \mu \mathrm{g} \mathrm{mL}^{-1}$. Primary antibody dilutions for immunostaining were mouse anti-GFP (Roche IgG1א clones 7.1 and 13.1) 1:200; rabbit SYM11 antibody (EMD Millipore, 07-413) 1:200; rabbit anti-Osk (a gift from P. Lasko) 1:1000;

Fig. 4 Wisp colocalizes and interacts with Aub. a, b Immunostaining of UASp-GFP-Aub nos-Gal4 stage 10 oocytes, also stained with DAPI (blue) (a) and 0-2 h-embryos (b), with anti-GFP and anti-Wisp. Posterior poles are shown in the bottom panels in $\mathbf{a}$, and in $\mathbf{b}$. Quantification of colocalization in the germ plasm using the Manders coefficient is shown in $\mathbf{b}$. Scale bars: $30 \mu \mathrm{m}$ in a top panels, and $10 \mu \mathrm{m}$ in $\mathbf{a}$ bottom panels and in $\mathbf{b}$. c Immunostaining of UASpGFP-Aub/nos-Gal4 0-2 h-embryos with anti-GFP and either anti-CCR4 (top panels), or anti-Smg (bottom panels). Scale bars: $10 \mu \mathrm{m}$. d Coimmunoprecipitation of Wisp with GFP-Aub in UASp-GFP-Aub/nos-Gal4 0-2 h-embryos. $w^{1118}$ 0-2 h-embryos were used as negative control (Mock). Immunoprecipitation was with anti-GFP (GFP IP) either in the presence (+) or the absence (-) of RNase A. e Co-immunoprecipitation of Aub with Wisp in wild-type, csul and tud mutant 0-2 h-embryos. Immunoprecipitation was with anti-Wisp in the presence of RNase A. Bound proteins were detected using western blots with anti-Wisp and anti-Aub; inputs correspond to protein extracts before IP in d, e. f GST pull-down assays between GST-Wisp and HAAub. Constructs and interactions are shown in the table. HA-tagged Aub fragments were revealed using western blot with anti-HA. Inputs correspond to 1:10 of in vitro-synthetized HA-Aub fragments before pull-down. GST alone was used as a negative control. GST and GST-recombinant proteins used in each pull-down are shown (bottom panels). Arrowheads indicate full-length recombinant proteins 
a
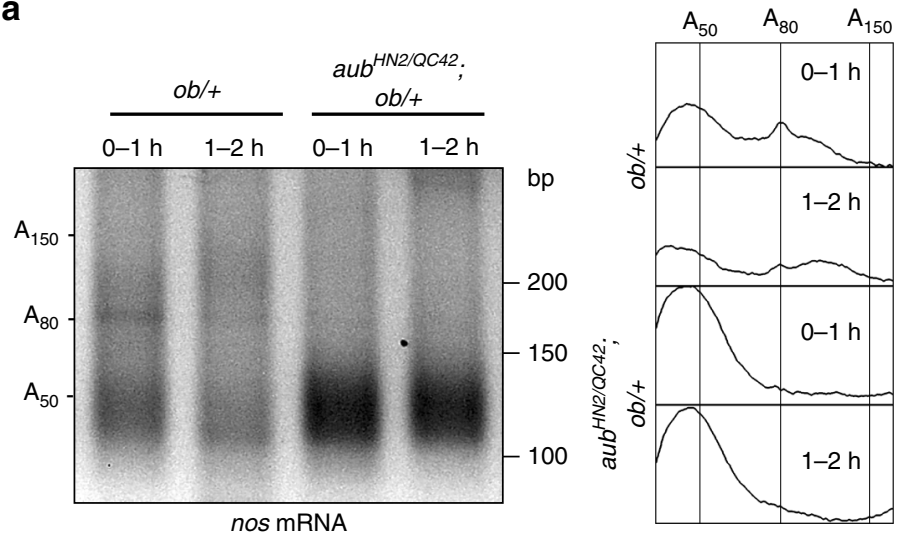

b
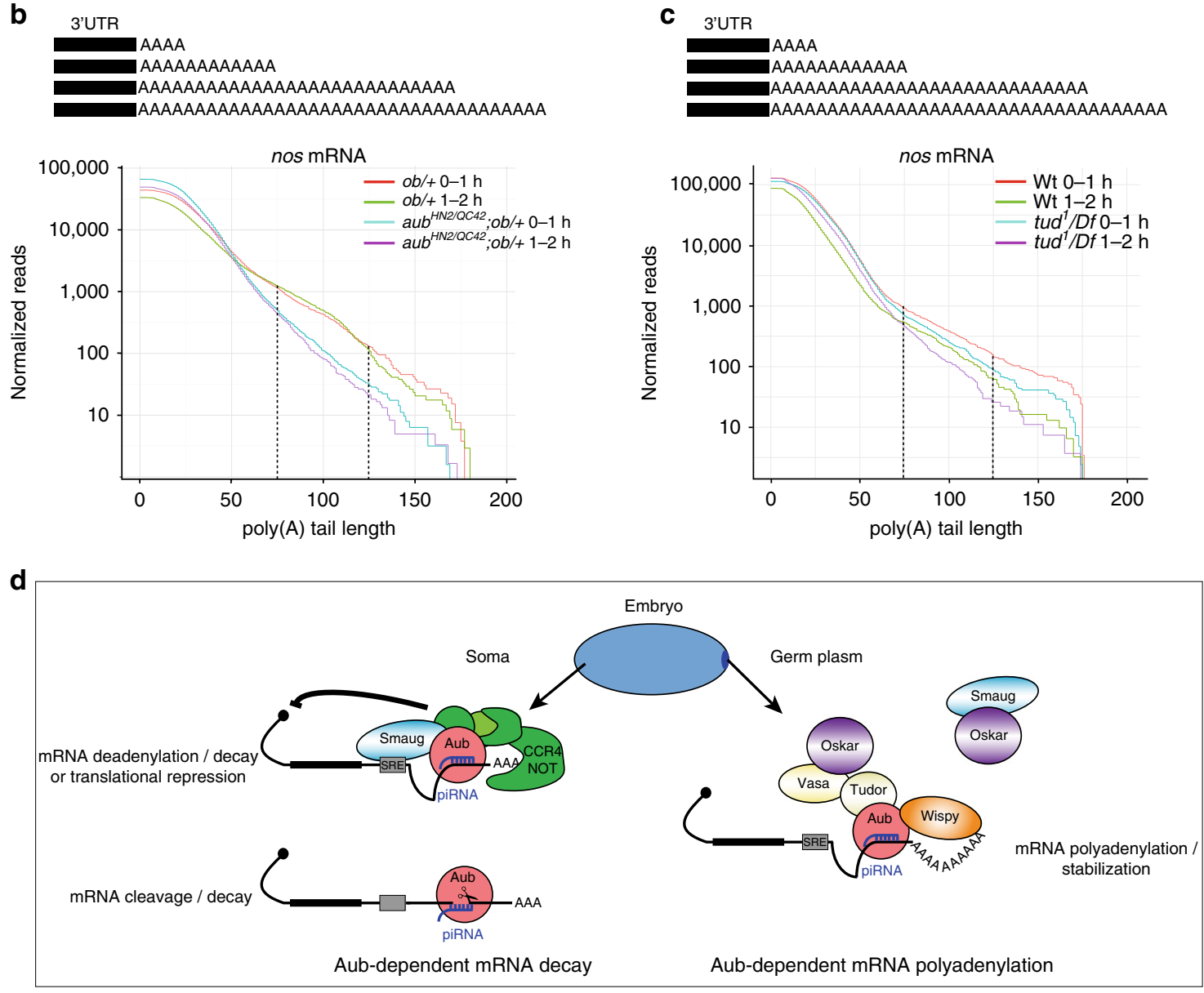

Fig. 5 Aub recruits Wisp to stabilize germ cell mRNAs in the germ plasm. a ePAT assays of nos mRNA in 0-1 h- and 1-2 h-ob/+ embryos, in wild-type and aub mutant backgrounds. ePAT assay profiles using ImageJ are shown on the right. b, c Distribution of sequenced nos mRNA poly(A) tails in ob/+ and $a u b^{-} ; o b /+$ mutant embryos (b), and in wild-type and tud mutant embryos (c). Each curve represents the mean of two biological replicates normalized to reads per million. The mPAT reads are displayed as cumulative plots: all reads having at least a specific number of non-templated A-bases are pooled, as shown in the scheme (top panel). The $x$-axis represents the number of non-templated A-bases sequenced at the end of each read and the $y$-axis represents the normalized number of reads on a log scale. The portion of the graph corresponding to the pool of nos mRNA with long poly(A) tails is indicated with broken lines. In $\mathbf{b}$, the proportion of reads having 100 A-bases or more (\% of total) is significantly reduced in the aub mutant ( $p=0.0025)$ by two-way ANOVA, which takes into consideration both time and genotype variables. $\mathbf{d}$ Model of the dual function of piRNA-loaded Aub in maternal mRNA somatic decay and stabilization in the germ plasm. See text for details

mouse anti-Aub $(4 \mathrm{D} 10)^{48}$ 1:800; rabbit anti-Aub (Abcam, ab17724) 1:100; mouse anti-Armi ${ }^{49}$ 1:500; mouse anti-Wispy (7B3, a gift from N. Kim) 1:100; guinea pig anti-Smg $^{50}$ 1:1000; and rabbit anti-CCR ${ }^{51} 1: 200$. Secondary antibodies (antirabbit Alexa 488-conjugated (Invitrogen, A-11034); anti-mouse Alexa 488conjugated (Invitrogen, A-11029); anti-rabbit Cyanine 3-conjugated (Jackson Immunoresearch, 711-165-148); anti-mouse Cyanine 3-conjugated (Jackson
Immunoresearch, 115-166-006); anti-mouse Alexa 647-conjugated (Invitrogen, A21236); anti-guinea pig Cyanine 3-conjugated (Jackson Immunoresearch, 706-165148)) were used at dilution 1:500. For whole-mount in situ hybridization experiments, fixed embryos were rehydrated gradually with methanol-PBT-Tween (PBS supplemented with $0.1 \%$ Tween-20) and washed three times in PBT-Tween. Embryos were prehybridized in pre-warm HB buffer (50\% formamide, $5 \times$ SSC, 50 
$\mu \mathrm{g} \mathrm{mL} \mathrm{m}^{-1}$ heparin, $0.1 \%$ Tween-20, $5 \mu \mathrm{g} \mathrm{mL}^{-1}$ torula yeast RNA (Sigma)) for $1 \mathrm{~h}$ at $65^{\circ} \mathrm{C}$ and hybridized with 1:50 to $1: 100$ of probe in $\mathrm{HB}$ buffer overnight at $65^{\circ} \mathrm{C}$. Embryos were washed for $15 \mathrm{~min}$ at $65^{\circ} \mathrm{C}$ twice with $2 \mathrm{X}$ SSC, $0.1 \%$ Tween-20, twice with $0.2 \times$ SSC, $0.1 \%$ Tween-20, and at room temperature three times with PBT-Tween. Embryos were incubated with preabsorbed anti-Digoxigenin-alcaline phosphatase antibody (Roche, 11093274910) at 1:2000 for $1.5 \mathrm{~h}$ at room temperature on a wheel. They were washed three times for $20 \mathrm{~min}$ in PBT-Tween and once for $20 \mathrm{~min}$ in AP buffer (100 mM Tris $2 \mathrm{M} \mathrm{pH} \mathrm{9.5,} 50 \mathrm{mM} \mathrm{MgCl}_{2}, 100 \mathrm{mM}$ $\mathrm{NaCl}, 0.1 \%$ Tween-20). Revelation was in $1 \mathrm{~mL}$ AP buffer with $7.5 \mu \mathrm{L}$ NBT (nitro blue tetrazolium) and 5.6 $\mu \mathrm{L}$ BCIP (5-bromo-4-chloro-3-indolyl-phosphate) for 5-15 min, and was stopped by three washes with PBT-Tween. Embryos were dehydrated gradually with ethanol-PBT-Tween and washed twice in ethanol $100 \%$. Mounting was in Canada Balsam supplemented with methyl salicylate (4:1). Probes for in situ hybridization experiments were Digoxigenin-labeled antisense RNA in vitro transcribed from coding regions of the corresponding genes cloned into the Topo TA pCRTM II vector (Invitrogen) ${ }^{11}$ or from the pN5 nos cDNA clone. For fluorescent in situ hybridization coupled with immunostaining (immuno-FISH), after hybridization and washing of the probe, embryos or ovaries were blocked $1 \mathrm{~h}$ in PBTHBR (1× PBS, 0.1\% Triton-X100, 0.04\% horse serum, $0.001 \%$ BSA, $40 \mathrm{U} \mu \mathrm{L}$ ${ }^{-1}$ RNase Inhibitor (Promega)) and incubated overnight at $4{ }^{\circ} \mathrm{C}$ in PBTHBR with anti-Digoxigenin-POD antibody (peroxidase-conjugated, Jackson Immunoresearch, 200-032-156) at 1:200 and other antibodies. Embryos or ovaries were washed three times for $20 \mathrm{~min}$ in PBT-Tween. Tyramide Signal Amplification was performed at room temperature on a wheel for $10 \mathrm{~min}$ with 1:25 TSA Cyanine 3 in amplification diluent provided by the manufacturer (TSA ${ }^{\circledR}$ Cyanine 3, PerkinElmer). The reaction was stopped with two quick washes in PBT-Tween, followed by three $10 \mathrm{~min}$-washes in PBT-Tween. Secondary antibodies were incubated at room temperature for $2 \mathrm{~h}$ and washed three times for $10 \mathrm{~min}$ in PBT-Tween. Mounting was in Vectashield.

Microscopy and image processing. Fluorescent images were acquired using a Zeiss LSM 780 laser scanning confocal microscope equipped with a Zeiss $40 \times$ PLAN-APO 1.3 oil-immersion DIC (UV) VIS-IR and a Zeiss 20× PLAN-APO 0.8 objective lens. The acquisition software was Zen. Contrast and relative intensities were processed and quantified with ImageJ software. Light microscope images were acquired using Leica Leitz DMRB Fluorescence-Phase Contrast Microscope with Nomarsky lens.

RT-qPCR and PAT assays. Total RNA was prepared from 30 embryos using Trizol (Invitrogen). RNA concentration was determined with nanodrop ND1000 spectrophotometer. For RT-qPCR, $0.5-1 \mu \mathrm{g}$ of total RNA was reverse transcribed with SuperScript III (Invitrogen) and random hexamers (Invitrogen). RNA levels were calculated using the LightCycler ${ }^{\circledR} 480$ SYBR Green I Master (Roche) on the LightCycler ${ }^{\circledR} 480$ Instrument (Roche) and the primers 5'-CGGAGCTTCCAA TTCCAGTAAC-3' and 5'-AGTTATCTCGCACTGAGTGGCT-3' for nos, and 5'CTGTGAGAGTTCGCCAAATG- ${ }^{\prime}$ and $5^{\prime}$-CATTGAGTTTCCGGTGTGTC- ${ }^{\prime}$ for RpL32. Poly(A) test (PAT) assays were performed with $1 \mu \mathrm{g}$ of total RNA using either regular PAT (Fig. 3), or ePAT (Fig. 5) methods ${ }^{52}$. For the PAT reaction, mRNA poly(A) tails were coated with $0.1 \mu$ g oligo- $\mathrm{d}(\mathrm{T})_{12-18}$ primers which were then ligated with $40 \mathrm{U}$ of T4 DNA Ligase; this reaction was followed by annealing of the $\mathrm{d}(\mathrm{T})$-anchor primer to the overhanging remaining As at $12{ }^{\circ} \mathrm{C}$ and its subsequent ligation, then by reverse transcription with SuperScript III (Invitrogen) from this ligated primer, and PCR using the primers 5'-GCGAGCTCCGCGGCCG CGTTTTTTTTTTTT-3' (d(T)-anchor) and 5'-TTTTGTTTACCATTGATCAATT TTTC-3' for nos or 5'-GGATTGCTACACCTCGGCCCGT-3' for sop. For ePAT, mRNA poly(A) tails was annealed with the $\mathrm{d}(\mathrm{T})$-anchor primer by mixing $1 \mu \mathrm{g}$ of total RNA with $2 \mu \mathrm{L}$ of $50 \mu \mathrm{M} \mathrm{d}(\mathrm{T})$-anchor primer and used as template for mRNA extension with $5 \mathrm{U}$ of DNA Polymerase I, Large (Klenow) Fragment (New England Biology) at $37^{\circ} \mathrm{C}$; this reaction was then switched to $55^{\circ} \mathrm{C}$ to dissociate annealings that had not been extended by DNA Polymerase I, and followed by reverse transcription with SuperScript III (Invitrogen) and PCR using d(T)-anchor and the specific primer $5^{\prime}$-GAAAAATTCAATGGCTCGAGTGCC-3' for nos. PCR fragments were visualized on $2 \%$ agarose gel.

MPAT. To improve the resolution and sensitivity of gel-based PAT assays, we adapted the ePAT approach ${ }^{53}$ to multiplexing on the Illumina MiSeq instrument. Sequencing ensures that any amplicon detected is specific to the gene of interest, and enables a digital read-out of the amplicon amount, and visualization of the distribution of sequenced poly(A) lengths. We refer to this assay as MPAT for multiplexed Poly(A) Test. A nested-PCR approach was used to sequentially incorporate the P5 and P7 elements necessary for bridge-amplification and sequencing on the Illumina flow-cell. First, ePAT cDNA was generated using the mPAT Reverse Primer 5'-CAGACGTGTGCTCTTCCGATCTTTTTTTTTTTTT$3^{\prime}$ using 500 ng total RNA from the indicated genotypes as input. In a first round of PCR amplification the sequence 5'-CCTACACGACGCTCTTCCGATCT- $3^{\prime}$ was appended upsteam of traditional PAT primers designed $\sim 100 \mathrm{nt}$ from the polyadenylation site: nos mPAT 5'-CCTACACGACGCTCTTCCGATCTCACACATG AAACAACCGCCA-3'; $p g c$ mPAT 5'-CCTACACGACGCTCTTCCGATCTCAAG AACAAGGAGGGAAGCTCG-3'; tim 10 mPAT 5'-CCTACACGACGCTCTTCCG
ATCTGCGCTACGATTGTTAGAGGTAC-3'. A pool of such gene-specific primers were used in five cycles of first-round amplification with the mPAT reverse primer. Unincorporated primers from this first round amplification were removed using NucleoSpin columns (Macherey-Nagel). Eluted amplicons were entered into a second round PCR using the universal Illumina Rd1 sequencing Primer 5'-AAT GATACGGCGACCACCGAGATCTACACTCTTTCCCTACACGACGCTCTTCC G-3' and TruSeq indexed reverse primers from Illumina, with ten cycles of amplification. Note that each experimental condition was amplified separately in the first round with identical pooled primers. In the second round, each experimental condition received a different indexing primer. These second-round PCR reactions were pooled, cleared of excess primers using AMPure XP beads (Beckman Coulter) and sequenced using the MiSeq Reagent Kit v2 with 300 cycles (i.e., 300 bases of sequencing) according to the manufacturer's specifications. The data were analyzed using established bioinformatics pipelines ${ }^{54}$ and figures were generated using the $\mathrm{R}$ framework.

Immunoprecipitations. For immunoprecipitations, $0-2$ h-embryos $(\approx 100 \mu \mathrm{L}$ embryos per IP) were homogenized in $500 \mu \mathrm{L}$ DXB-150 $(25 \mathrm{mM}$ Hepes- $\mathrm{KOH}$ pH 6.8, $250 \mathrm{mM}$ sucrose, $1 \mathrm{mM} \mathrm{MgCl}_{2}, 1 \mathrm{mM}$ DTT, $150 \mathrm{mM} \mathrm{NaCl}, 0.1 \%$ Triton X-100) containing cOmplete ${ }^{\mathrm{TM}}$ EDTA-free Protease Inhibitor Cocktail (Roche) and either RNase Inhibitor $\left(0.25 \mathrm{U}^{-1}\right.$, Promega) or RNase A $\left(2 \mu \mathrm{g} \mathrm{mL} \mathrm{L}^{-1}\right.$, Sigma). A total of $50 \mu \mathrm{L}$ Dynabeads protein A (Invitrogen) were incubated with either $10 \mu \mathrm{L}$ mouse anti-GFP (monoclonal antibody 3E6, Invitrogen, A-11120), $5 \mu \mathrm{L}$ mouse anti-Wisp (14D1, a gift from N. Kim), or $5 \mu \mathrm{L}$ purified mouse IgG (Invitrogen, 02-6502) (mock IP) for $1 \mathrm{~h}$ on a wheel at room temperature. Protein extracts were cleared on $30 \mu \mathrm{L}$ Dynabeads protein A previously equilibrated with DXB-150 for $30 \mathrm{~min}$ at $4{ }^{\circ} \mathrm{C}$. The pre-cleared protein extracts were incubated with Dynabeads protein A bound to antibodies for $3 \mathrm{~h}$ at $4^{\circ} \mathrm{C}$. The beads were then washed 7 times with DXB150 for $10 \mathrm{~min}$ at room temperature. Proteins were eluted in 1X NUPAGE buffer supplemented with $100 \mathrm{mM}$ DTT at $70^{\circ} \mathrm{C}$ and analyzed using western blots with antibodies at the following dilutions: mouse anti-Aub (4D10) ${ }^{48}$ 1:2500; rabbit antiAub (Abcam, ab17724) 1:2000; guinea pig anti-Wispy ${ }^{30}$ 1:3000; rabbit antiWispy $^{31}$ 1:2500. Complete blots are shown in Supplementary Fig. 6.

GST pull-down assays. The constructs for production of GST-Wisp(11-547) and GST-Wisp(702-1373) were previously generated ${ }^{30}$. The N-terminal half of Wisp (amino acids 1-713) was cloned into the pGEX-5X-2 vector using NotI and XhoI The central region of Wisp (amino acids 636-746) was amplified by PCR and cloned into the pGEX-4T-1 vector, digested with EcoRI and XhoI. HA-Aub constructs were obtained by cloning PCR-amplified Aub fragments (amino acids 1-482 and 476-866) into the EcoRI and XhoI sites of the pCSH2 vector (pCS2 + backbone vector with two HA tags). GST-fused proteins were expressed in E. coli BL21 and affinity-purified on glutathione-Sepharose 4B beads (GE Healthcare); the beads were incubated overnight at $4{ }^{\circ} \mathrm{C}$ in PBT, cOmplete ${ }^{\mathrm{TM}}$ EDTA-free Protease Inhibitor Cocktail (Roche) and 5\% BSA. HA-tagged proteins were synthesized in vitro using the TnT Coupled reticulocyte lysate system (Promega). HA-tagged proteins were incubated with immobilized GST fusion proteins for $1.5 \mathrm{~h}(45 \mathrm{~min}$ at room temperature followed by $45 \mathrm{~min}$ at $4{ }^{\circ} \mathrm{C}$ ) in $400 \mu$ l binding buffer (50 mM Hepes pH 7.5, $600 \mathrm{mM} \mathrm{NaCl}, 0.2 \mathrm{mM}$ EDTA, $1 \mathrm{mM}$ DTT, $0.5 \%$ Nonidet P-40, cOmplete ${ }^{\mathrm{TM}}$ EDTA-free Protease Inhibitor Cocktail (Roche)) containing $0.2 \mu \mathrm{g} \mu \mathrm{L}^{-1}$ RNase A. Beads were washed four times with binding buffer. Recom binant proteins were dissociated from the beads by boiling $5 \mathrm{~min}$ in Laemmli buffer and separated on a SDS-PAGE gel. Western blots were revealed with mouse antiHA antibody (Covance, MMS-101R) at dilution 1:1000.

Statistical and bioinformatic analyses. Colocalization was analyzed using the ImageJ tool Coloc2 with 4-5 embryos or oocytes and calculated using the Manders's overlap coefficient ${ }^{55}$. Prediction of piRNA target sites on cellular mRNAs was performed as follows. We used a pool of piRNAs from 0-2 h-embryos sequenced in previously published libraries (GSM327625, GSM327626, GSM327627, GSM327628, GSM327629, GSM1818089, GSM1818091). This led to a total of 3,305,903 non-redundant piRNA sequences. Bowtie was used with different complementarities to identify piRNA target sites on transcripts with reproduced cross-links, as follows. Bowtie with option '-v 0', '-v1', '-v 2' or '-v3' was used to identify piRNAs that potentially target mRNAs with up to $0,1,2$ or 3 mismatch (es), respectively. For complementarities with a seed, we did not use quality values, therefore the sum of the quality values at all mismatched read positions (-e/maqerr) was set to an arbitrary value of 2000 , which disabled the quality values. Furthermore, -l (length of the seed) and -n (number of mismatches within the seed) were set to different values. The option '-nofw' was used to search only for reverse-complementarity between piRNAs and mRNAs.

Data availability. Accession numbers of previously published datasets are GSM327625, GSM327626, GSM327627, GSM327628, GSM327629, GSM1818089, GSM1818091. mPAT sequences generated in this study have been deposited to figshare, under the link: https://doi.org/10.4225/03/59b074beaefcc 
Received: 10 January 2017 Accepted: 18 September 2017

Published online: 03 November 2017

\section{References}

1. Voronina, E., Seydoux, G., Sassone-Corsi, P. \& Nagamori, I. RNA granules in germ cells. Cold Spring Harb. Perspect. Biol. 3, doi:https://doi.org/10.1101/ cshperspect.a002774 (2011).

2. Martin, K. C. \& Ephrussi, A. mRNA localization: gene expression in the spatial dimension. Cell 136, 719-730 (2009).

3. Forrest, K. M. \& Gavis, E. R. Live imaging of endogenous RNA reveals a diffusion and entrapment mechanism for nanos mRNA localization in Drosophila. Curr. Biol. 13, 1159-1168 (2003).

4. Sinsimer, K. S., Jain, R. A., Chatterjee, S. \& Gavis, E. R. A late phase of germ plasm accumulation during Drosophila oogenesis requires lost and rumpelstiltskin. Development 138, 3431-3440 (2011).

5. Bergsten, S. E. \& Gavis, E. R. Role for mRNA localization in translational activation but not spatial restriction of nanos RNA. Development 126, 659-669 (1999).

6. Trcek, T. et al. Drosophila germ granules are structured and contain homotypic mRNA clusters. Nat. Commun. 6, 7962 (2015).

7. Zaessinger, S., Busseau, I. \& Simonelig, M. Oskar allows nanos mRNA translation in Drosophila embryos by preventing its deadenylation by Smaug/ CCR4. Development 133, 4573-4583 (2006).

8. Thomson, T., Liu, N., Arkov, A., Lehmann, R. \& Lasko, P. Isolation of new polar granule components in Drosophila reveals P body and ER associated proteins. Mech. Dev. 125, 865-873 (2008).

9. Guzzardo, P. M., Muerdter, F. \& Hannon, G. J. The piRNA pathway in flies: highlights and future directions. Curr. Opin. Genet. Dev. 23, 44-52 (2013).

10. Ishizu, H., Siomi, H. \& Siomi, M. C. Biology of PIWI-interacting RNAs: new insights into biogenesis and function inside and outside of germlines. Genes Dev. 26, 2361-2373 (2012).

11. Barckmann, B. et al. Aubergine iCLIP reveals piRNA-dependent decay of mrnas involved in germ cell development in the early embryo. Cell Rep. 12, 1205-1216 (2015).

12. Goh, W. S. et al. piRNA-directed cleavage of meiotic transcripts regulates spermatogenesis. Genes Dev. 29, 1032-1044 (2015).

13. Gou, L. T. et al. Pachytene piRNAs instruct massive mRNA elimination during late spermiogenesis. Cell. Res. 24, 680-700 (2014).

14. Kiuchi, T. et al. A single female-specific piRNA is the primary determiner of sex in the silkworm. Nature 509, 633-636 (2014).

15. Rouget, C. et al. Maternal mRNA deadenylation and decay by the piRNA pathway in the early Drosophila embryo. Nature 467, 1128-1132 (2010).

16. Watanabe, T., Cheng, E. C., Zhong, M. \& Lin, H. Retrotransposons and pseudogenes regulate mRNAs and $\operatorname{lncRNAs}$ via the piRNA pathway in the germline. Genome Res. 25, 368-380 (2015).

17. Zhang, P. et al. MIWI and piRNA-mediated cleavage of messenger RNAs in mouse testes. Cell. Res. 25, 193-207 (2015).

18. Rangan, P. et al. Temporal and spatial control of germ-plasm RNAs. Curr. Biol. 19, 72-77 (2009).

19. Malone, C. D. et al. Specialized piRNA pathways act in germline and somatic tissues of the Drosophila ovary. Cell 137, 522-535 (2009).

20. Klattenhoff, C. et al. Drosophila rasiRNA pathway mutations disrupt embryonic axis specification through activation of an ATR/Chk2 DNA damage response. Dev. Cell. 12, 45-55 (2007).

21. Pane, A., Wehr, K. \& Schupbach, T. zucchini and squash encode two putative nucleases required for rasiRNA production in the Drosophila germline. Dev. Cell. 12, 851-862 (2007).

22. Ephrussi, A. \& Lehmann, R. Induction of germ cell formation by oskar. Nature 358, 387-392 (1992).

23. Wilson, J. E., Connell, J. E. \& Macdonald, P. M. aubergine enhances oskar translation in the Drosophila ovary. Development 122, 1631-1639 (1996).

24. Gavis, E. R. \& Lehmann, R. Localization of nanos RNA controls embryonic polarity. Cell 71, 301-313 (1992).

25. Vourekas, A., Alexiou, P., Vrettos, N., Maragkakis, M. \& Mourelatos, Z. Sequence-dependent but not sequence-specific piRNA adhesion traps mRNAs to the germ plasm. Nature 531, 390-394 (2016).

26. Mohn, F., Handler, D. \& Brennecke, J. Noncoding RNA. piRNA-guided slicing specifies transcripts for Zucchini-dependent, phased piRNA biogenesis. Science 348, 812-817 (2015).

27. Kirino, Y. et al. Arginine methylation of Piwi proteins catalysed by dPRMT5 is required for Ago3 and Aub stability. Nat. Cell. Biol. 11, 652-658 (2009).

28. Nishida, K. M. et al. Functional involvement of Tudor and dPRMT5 in the piRNA processing pathway in Drosophila germlines. EMBO. J. 28, 3820-3831 (2009).
29. Kirino, Y. et al. Arginine methylation of Aubergine mediates Tudor binding and germ plasm localization. RNA 16, 70-78 (2010).

30. Benoit, P., Papin, C., Kwak, J. E., Wickens, M. \& Simonelig, M. PAP- and GLD2-type poly(A) polymerases are required sequentially in cytoplasmic polyadenylation and oogenesis in Drosophila. Development 135, 1969-1979 (2008).

31. Cui, J., Sackton, K. L., Horner, V. L., Kumar, K. E. \& Wolfner, M. F. Wispy, the Drosophila homolog of GLD-2, is required during oogenesis and egg activation. Genetics 178, 2017-2029 (2008).

32. Cui, J., Sartain, C. V., Pleiss, J. A. \& Wolfner, M. F. Cytoplasmic polyadenylation is a major mRNA regulator during oogenesis and egg activation in Drosophila. Dev. Biol. 383, 121-131 (2013).

33. Eichhorn, S. W. et al. mRNA poly(A)-tail changes specified by deadenylation broadly reshape translation in Drosophila oocytes and early embryos. eLife 5, e16955 (2016).

34. Lim, J., Lee, M., Son, A., Chang, H. \& Kim, V. N. mTAIL-seq reveals dynamic poly(A) tail regulation in oocyte-to-embryo development. Genes Dev. 30, 1671-1682 (2016).

35. Thomsen, S., Anders, S., Janga, S. C., Huber, W. \& Alonso, C. R. Genome-wide analysis of mRNA decay patterns during early Drosophila development. Genome. Biol. 11, R93 (2010).

36. Becalska, A. N. et al. Aubergine is a component of a nanos mRNA localization complex. Dev. Biol. 349, 46-52 (2011).

37. Dahanukar, A., Walker, J. A. \& Wharton, R. P. Smaug, a novel RNA-binding protein that operates a translational switch in Drosophila. Mol. Cell. 4, 209-218 (1999).

38. Wang, L., Eckmann, C. R., Kadyk, L. C., Wickens, M. \& Kimble, J. A regulatory cytoplasmic poly(A) polymerase in Caenorhabditis elegans. Nature 419, 312-316 (2002).

39. Schupbach, T. \& Wieschaus, E. Female sterile mutations on the second chromosome of Drosophila melanogaster. II. Mutations blocking oogenesis or altering egg morphology. Genetics 129, 1119-1136 (1991).

40. Cook, H. A., Koppetsch, B. S., Wu, J. \& Theurkauf, W. E. The Drosophila SDE3 homolog armitage is required for oskar mRNA silencing and embryonic axis specification. Cell 116, 817-829 (2004).

41. Abdu, U., Brodsky, M. \& Schupbach, T. Activation of a meiotic checkpoint during Drosophila oogenesis regulates the translation of Gurken through Chk2/ Mnk. Curr. Biol. 12, 1645-1651 (2002).

42. Schupbach, T. \& Wieschaus, E. Germline autonomy of maternal-effect mutations altering the embryonic body pattern of Drosophila. Dev. Biol. 113, 443-448 (1986).

43. Parks, A. L. et al. Systematic generation of high-resolution deletion coverage of the Drosophila melanogaster genome. Nat. Genet. 36, 288-292 (2004).

44. Anne, J., Ollo, R., Ephrussi, A. \& Mechler, B. M. Arginine methyltransferase Capsuleen is essential for methylation of spliceosomal Sm proteins and germ cell formation in Drosophila. Development 134, 137-146 (2007).

45. Murata, Y. \& Wharton, R. P. Binding of pumilio to maternal hunchback mRNA is required for posterior patterning in Drosophila embryos. Cell 80, 747-756 (1995).

46. Rorth, P. Gal4 in the Drosophila female germline. Mech. Dev. 78, 113-118 (1998).

47. Harris, A. N. \& Macdonald, P. M. Aubergine encodes a Drosophila polar granule component required for pole cell formation and related to eIF2C. Development 128, 2823-2832 (2001).

48. Gunawardane, L. S. et al. A slicer-mediated mechanism for repeat-associated siRNA 5' end formation in Drosophila. Science 315, 1587-1590 (2007).

49. Saito, K. et al. Roles for the $\mathrm{Yb}$ body components Armitage and $\mathrm{Yb}$ in primary piRNA biogenesis in Drosophila. Genes Dev. 24, 2493-2498 (2010).

50. Tadros, W. et al. SMAUG is a major regulator of maternal mRNA destabilization in Drosophila and its translation is activated by the PAN GU kinase. Dev. Cell. 12, 143-155 (2007).

51. Temme, C., Zaessinger, S., Meyer, S., Simonelig, M. \& Wahle, E. A complex containing the CCR4 and CAF1 proteins is involved in mRNA deadenylation in Drosophila. EMBO J. 23, 2862-2871 (2004).

52. Chartier, A., Joly, W. \& Simonelig, M. Measurement of mRNA Poly(A) Tail lengths in drosophila female germ cells and germ-line stem cells. Methods Mol. Biol. 1463, 93-102 (2017).

53. Janicke, A., Vancuylenberg, J., Boag, P. R., Traven, A. \& Beilharz, T. H. ePAT: a simple method to tag adenylated RNA to measure poly(A)-tail length and other 3' RACE applications. RNA 18, 1289-1295 (2012).

54. Harrison, P. F. et al. PAT-seq: a method to study the integration of $3^{\prime}$-UTR dynamics with gene expression in the eukaryotic transcriptome. RNA 21, 1502-1510 (2015).

55. Manders, E. M., Verbeek, F. J. \& Aten, J. A. Measurement of co-localization of objects in dual-colour confocal images. J. Microsc. 169, 375-382 (1993). 


\section{Acknowledgements}

This work is dedicated to the memory of our friend and colleague Cathy Papin. We thank P. Lasko, N. Kim, M. Siomi and M. Wolfner for their gifts of antibodies, and A. Ephrussi, E. Gavis, P. MacDonald and W. Theurkauf for their gifts of fly stocks. We thank M. Curtis, A. Barugahare, Micromon and the Monash Bioinformatic Platform for technical support. This work was supported by UMR9002 CNRS-University of Montpellier, ANR (ANR-2010-BLAN-1201 01 and ANR-15-CE12-0019-01), FRM ("Equipe FRM 2013 DEQ20130326534" and "Projets Innovants ING20101221078") and Fondation ARC (ARC Libre 2009, N³192). J.D. was supported by Fondation ARC and ANR, G.B. by Fondation ARC and FRM and C.J. by the Labex EpiGenMed (ANR-10LABX-12-01). S.P. held a salary from FRM "Projets Innovants" and "Equipe FRM 2013". T.H.B. was supported by a Biodiscovery Fellowship from Monash University.

\section{Author contributions}

M.S. and C.P.: Conceived the study; J.D., G.B., A.C., C.J., A.-C.M., C.P., M.S.: Performed experiments and analyzed the data. S.P.: Performed bioinformatic analyses. T.H.B. and P.F.H.: Performed mPAT and mPAT bioinformatic treatment, respectively. M.S.: wrote the manuscript with assistance from J.D. All authors discussed the manuscript.

\section{Additional information}

Supplementary Information accompanies this paper at doi:10.1038/s41467-017-01431-5.
Competing interests: The authors declare no competing financial interests.

Reprints and permission information is available online at http://npg.nature.com/ reprintsandpermissions/

Publisher's note: Springer Nature remains neutral with regard to jurisdictional claims in published maps and institutional affiliations.

(c) (i) Open Access This article is licensed under a Creative Commons Attribution 4.0 International License, which permits use, sharing, adaptation, distribution and reproduction in any medium or format, as long as you give appropriate credit to the original author(s) and the source, provide a link to the Creative Commons license, and indicate if changes were made. The images or other third party material in this article are included in the article's Creative Commons license, unless indicated otherwise in a credit line to the material. If material is not included in the article's Creative Commons license and your intended use is not permitted by statutory regulation or exceeds the permitted use, you will need to obtain permission directly from the copyright holder. To view a copy of this license, visit http://creativecommons.org/ licenses/by/4.0/.

(C) The Author(s) 2017 